Copyright (C 2014 IEEE. Personal use of this material is permitted. Permission from IEEE must be obtained for all other uses, in any current or future media, including reprinting/republishing this material for advertising or promotional purposes, creating new collective works, for resale or redistribution to servers or lists, or reuse of any copyrighted component of this work in other works. 


\title{
Electromechanical Piezoelectric Power Harvester Frequency Response Modelling Using Closed-Form Boundary Value Methods
}

\author{
Mikail F. Lumentut, Member, IEEE, and Ian M. Howard
}

\begin{abstract}
The conversion of mechanical vibration to electrical energy has shown great promise for extending battery life of smart sensor wireless devices for various engineering applications. This paper presents novel analytical models of a piezoelectric bimorph, using the closed-form boundary value (CFBV) method, for predicting the electromechanical power harvester frequency response. The derivations of the coupled electromechanical dynamic response of the transverse-longitudinal (CEDRTL) form based on the CFBV method were developed using the reduced strong form method of the Hamiltonian principle. The equations from CEDRTL can be reduced to give the coupled electromechanical dynamic response of the transverse (CEDRT) form. The electromechanical frequency response functions with variable load resistance were also given in detail using Laplace transformation. The two theoretical studies are compared together and validated with an experimental study. For some cases, when the load resistance approached open circuit, the difference between CEDRTL and CEDRT tended to be more pronounced. Conversely, the CEDRTL and CEDRT models tended to overlap when the load resistance approached short circuit. Nyquist plots are used to demonstrate the shifting frequency and amplitude changes due to variable resistance. Overall, the experimental and CEDRTL model results were very close to each other.
\end{abstract}

Index Terms - closed-form boundary value, electromechanical, energy harvesting, frequency, Hamiltonian, Nyquist plots, piezoelectric, smart sensor.

\section{INTRODUCTION}

$\mathrm{T}$ HE increasing demand of micropower harvesters is inevitable since there are growing autonomous sensor network devices that require self-sustained energy. This condition spurs the development of new methods for power capture and storage using rechargeable batteries for powering these devices. There are many examples of unused mechanical vibration energy that can potentially be converted into useful electrical energy such as in bridges, pipelines, industrial machinery and dynamic response of the human body. The viability of piezoelectric transduction with base vibration provides good promise for power harvesting technology with

Manuscript received on 16 December 2011, revised on 1 June 2012, accepted on 5 September 2012. Authors are with Theoretical and Applied Mechanics, Department of Mechanical Engineering, Curtin University, Australia (e-mails: mikail_lumentut@yahoo.com (corresponding author) and i.howard@exchange.curtin.edu.au). application to smart wireless sensor devices. The extensive reviews of the electromechanical piezoelectric-based power harvester literature have defined the key issues to be physical parameters such as design geometry, physical properties, power optimization, environmental mechanical energy, power conditioning electronic circuits and sensor systems [1]-[5]. Since electromechanical piezoelectric power harvesting is reliant upon physical aspects of the design geometry and its boundary conditions, research effort focusing on the experimental and analytical studies plays an important role for predicting the frequency response of the power output and its amplitude. Simplified analytical lumped parameter models with typical mass-spring-damper and electromechanical system have been used to formulate the electrical equivalent and power frequency response of the transverse bending piezoelectric beam [6] and the microcantilevered piezoelectric system with interdigital shaped electrodes [7]. Impedance matching of interface circuits have been used with a synchronized switch harvest on inductor (SSHI) for modelling single mode electrical equivalent systems representing the piezoelectric beam [8]. An electrical equivalent model of a fabricated PZT bimorph for optimising piezoelectric material constants, mechanical quality factor and electricity generation has also been developed [9]. Although the solution model using lumped parameter techniques attempted to provide a systems approach with various aspects of electromechanical power harvester dynamic response, it ignored the important physical issue of eigenfunction, convergence criteria and continuity. Moreover, a fabricated micro PMN-PT piezoelectric cantilever beam with electrode pattern and tuneable tip was used to develop the analytical elastic vibration model including the direct piezoelectric effect [10]. However, their analytical model ignored the backward piezoelectric coupling which can significantly affect the system electromechanical behaviour. Wickensheir et al. [11] discussed a standard electrical interface model of an AC-DC rectifier and storage capacitor for modelling time-varying input vibrations during the charging process using a single mode analytical piezoelectric beam.

Further analytical approaches using the Rayleigh-Ritz's method obtained a condensed matrix equation to analyse power harvesting frequency response with various load impedances [12], [13] where their methods were again limited to only a single mode of frequency response. With the same analytical approach, various aspects of resonance frequency 
behaviour of a unimorph piezoelectric beam were conducted by considering the truncation and tip mass ratios [14]. Moreover, Ly et al. [15] proposed an analytical model using the modal decomposition technique of the cantilevered EulerBernoulli piezoelectric beam with single layer to formulate transfer functions of the coupled electromechanical response system. The constitutive electromechanical finite element equations of Love-Kirchhoff's piezoelectric plate structure under harmonic and impact input excitations was also formulated and programmed in Matlab code to give the eigenmode shape, transient response FFT and frequency response [16], [17]. The analytical methods using weak and strong forms of electromechanical piezoelectric bimorph beams under two input dynamic excitations were formulated according to the Hamiltonian principle giving the multimode frequency responses. The analytical methods of the normalised Ritz eigenfunction and closed forms, reduced from weak and strong form respectively, were achieved with good agreement including the experimental validation [18]-[20].

In this paper, the analytical and experimental comparisons of the piezoelectric bimorph electromechanical dynamic responses under input base excitations are presented. The typical Euler-Bernoulli piezoelectric bimorph micropower harvester with tip mass was based on the PZT class with the plane-stress relationship, 3-1 mode of piezoelectric constant operation and 3-3 effect of piezoelectric permittivity. The reduced equations have been extended from the previous work [19]. In the present work, the authors present the new derivations of electromechanical frequency response functions using the CFBV methods. The CEDRTL model provided three normalised electromechanical dynamic equations whereas CEDRT gave two normalised electromechanical dynamic equations. These analytical studies were derived according to the CFBV equations reduced from the strong form of Hamiltonian's principle. In validation with the experimental study, the two theoretical methods are compared in order to investigate the one closest to the experimental response. In the forthcoming section, only the CEDRTL model was derived since this model can directly be simplified to obtain the CEDRT model. Moreover, examples of frequency response functions (FRFs) of the bimorph under the input base transverse acceleration using two analytical studies were validated using the tip absolute dynamic displacement, electrical voltage, current and power harvesting. The Nyquist frequency response plots were also analysed in order to further identify the amplitude and frequency response behaviours due to mechanical, electromechanical and resistive shunt dampings.

\section{ELECTROMECHANICAL DYNAMIC EQUATIONS WITH CEDRTL MODEL}

A piezoelectric bimorph beam with centre brass shim (substructure) was modelled here with input base transverse and longitudinal excitations. In this case, the strain energy from mechanical substructure response, electrical enthalpy, and kinetic energies of the bimorph and tip mass were used to formulate the constitutive electromechanical dynamic equa- tions of the piezoelectric bimorph using the strong form of Hamiltonian's principle to give the CFBV form [19]. The Piezoelectric bimorph model can be shown in Fig. 1. The reduced normalised CERDTL model based on CFBV can be formulated after simplifying [19], to give,

$$
\begin{gathered}
\ddot{u}_{r}(t)+2 \zeta_{r}^{(u)} \omega_{r}^{(u)} \dot{u}_{r}(t)+\omega_{r}^{(u)^{2}} u_{r}(t)+P_{r}^{(u)} v(t)=-Q_{r}^{(u)} \ddot{u}_{\text {base }}(t), \\
\ddot{w}_{r}(t)+2 \zeta_{r}^{(w)} \omega_{r}^{(w)} \dot{w}_{r}(t)+\omega_{r}^{(w)}{ }^{2} w(t)+P_{r}^{(w)} v(t)=-Q_{r}^{(w)} \ddot{w}_{\text {base }}(t), \\
\sum_{r=1}^{\infty} \hat{P}_{r}^{(u)} \dot{u}_{r}(t)+\sum_{r=1}^{\infty} \hat{P}_{r}^{(w)} \dot{w}_{r}(t)+P_{D} \dot{v}(t)+R_{L} v(t)=0 .
\end{gathered}
$$

It is noted that because (1) has been normalised, the parameters $P_{r}^{(u)}, P_{r}^{(w)}, \hat{P}_{r}^{(u)}, \hat{P}_{r}^{(w)}, P_{D}, R_{L}, Q_{r}^{(u)}$ and $Q_{r}^{(w)}$ can be reduced as,

$$
\begin{gathered}
P_{r}^{(u)}=-\hat{\vartheta}^{(G)} \hat{\Theta}_{r}(L), P_{r}^{(w)}=\hat{\vartheta}^{(H)} \frac{\mathrm{d} \hat{\Psi}_{r}(L)}{\mathrm{d} x}, \\
P_{D}=\int_{0}^{L} \hat{C}_{p} \mathrm{~d} x, \quad \sum_{r=1}^{\infty} \hat{P}_{r}^{(u)}=\sum_{r=1}^{\infty} \int_{0}^{L} \hat{\vartheta}^{(G)} \frac{\mathrm{d} \hat{\Theta}_{r}(x)}{\mathrm{d} x} \mathrm{~d} x, \\
\sum_{r=1}^{\infty} \hat{P}_{r}^{(w)}=-\sum_{r=1}^{\infty} \int_{0}^{L} \hat{\vartheta}^{(H)} \frac{\mathrm{d}^{2} \hat{\Psi}_{r}(x)}{\mathrm{d} x^{2}} \mathrm{~d} x, R_{L}=\frac{1}{R_{\text {load }}}, \\
Q_{r}^{(u)}=\int_{0}^{L} \hat{I}^{(A)} \hat{\Theta}_{r}(x) \mathrm{d} x+I_{t i p}^{(A)} \hat{\Theta}_{r}(L), Q_{r}^{(w)}=\int_{0}^{L} \hat{I}^{(A)} \hat{\Psi}_{r}(x) \mathrm{d} x+I_{\text {tip }}^{(A)} \hat{\Psi}_{r}(L) .
\end{gathered}
$$

The variables $u_{r}, w_{r}, v, u_{\text {base }}$ and $w_{\text {base }}$ indicate the generalised time dependent longitudinal and transverse coordinates, voltage, and longitudinal and transverse base excitations, respectively. Moreover, the coefficients $\hat{\vartheta}^{(G)}, \hat{\vartheta}^{(H)}, \hat{C}_{p}, \hat{I}^{(A)}$, $I_{t i p}^{(A)}, \hat{\Theta}_{r}($.$) and \hat{\Psi}_{r}($.$) indicate the longitudinal and transverse$ piezoelectric couplings, piezoelectric capacitance, zeroth mass moment of inertia of the bimorph and tip mass, and normalised mode shapes of longitudinal and transverse forms, respectively, as expressed in Appendices A, B, C, and D where the piezoelectric parameters are written in accordance with IEEE standards [21]. Damping ratios $\zeta_{r}^{(u)}$ and $\zeta_{r}^{(w)}$ including load resistance $R_{\text {load }}$ are further discussed in Section IV. It is important to note here that the CEDRT model can be formulated by simply using (1) and ignoring the electromechanical longitudinal form to give,

$$
\begin{gathered}
\ddot{w}_{r}(t)+2 \zeta_{r}^{(w)} \omega_{r}^{(w)} \dot{w}_{r}(t)+\omega_{r}^{(w)^{2}} w(t)+P_{r}^{(w)} v(t)=-Q_{r}^{(w)} \ddot{w}_{\text {base }}(t), \\
\sum_{r=1}^{\infty} \hat{P}_{r}^{(w)} \dot{w}_{r}(t)+P_{D} \dot{v}(t)+R_{L} v(t)=0 .
\end{gathered}
$$

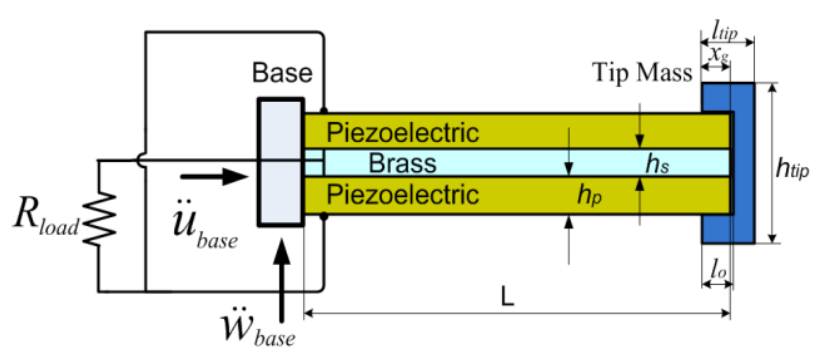

Fig. 1. Piezoelectric bimorph beam with a tip mass. 
It is noted that equation (1) and (3) can be further formulated using Laplace transformation. However, in this paper, we further retain and formulate the CEDRTL model since it gives particular insight of the multielectromechanical responses and provides more complete mathematical treatment compared with the CEDRT model. Equation (1) can be solved using Laplace transformations, where the multimode electromechanical dynamic equations of the piezoelectric bimorph can be reduced as,

$$
\begin{aligned}
& u_{r}(s)=-\frac{1}{Z(s)_{r}}\left[\left\{\left(s^{2}+2 \zeta_{r}^{(w)} \omega_{r}^{(w)} s+\omega_{r}^{(w)^{2}}\right)\left(s P_{D}+R_{L}\right)-\sum_{r=1}^{\infty} s \hat{P}_{r}^{(w)} P_{r}^{(w)}\right\}\right. \\
& \left.\times Q_{r}^{(u)} s^{2} u_{\text {base }}(s)+\sum_{r=1}^{\infty} s \hat{P}_{r}^{(w)} P_{r}^{(u)} Q_{r}^{(w)}\left(s^{2} w_{\text {base }}(s)\right)\right] \text {, } \\
& w_{r}(s)=-\frac{1}{Z(s)_{r}}\left[\left\{\left(s^{2}+2 \zeta_{r}^{(u)} \omega_{r}^{(u)} s+\omega_{r}^{(u)^{2}}\right)\left(s P_{D}+R_{L}\right)-s \sum_{r=1}^{\infty} \hat{P}_{r}^{(u)} P_{r}^{(u)}\right\}\right. \\
& \left.\times Q_{r}^{(w)} s^{2} w_{\text {base }}(s)+\sum_{r=1}^{\infty} s \hat{P}_{r}^{(u)} P_{r}^{(w)} Q_{r}^{(u)} s^{2} u_{\text {base }}(s)\right], \\
& v(s)=\frac{1}{Z(s)_{r}}\left[\sum_{r=1}^{\infty}\left\{s \hat{P}_{r}^{(w)} Q_{r}^{(w)}\left(s^{2}+2 \zeta_{r}^{(u)} \omega_{r}^{(u)} s+\omega_{r}^{(u)^{2}}\right) s^{2} w_{\text {base }}(s)\right\}\right. \\
& \left.+\sum_{r=1}^{\infty}\left\{-s \hat{P}_{r}^{(u)} Q_{r}^{(u)}\left(s^{2}+2 \zeta_{r}^{(w)} \omega_{r}^{(w)} s+\omega_{r}^{(w)^{2}}\right) s^{2} u_{\text {base }}(s)\right\}\right] .
\end{aligned}
$$

The characteristic polynomial form from (4) to (6) can be expressed as,

$$
\begin{aligned}
Z(s)_{r}= & \left(s^{2}+2 \zeta_{r}^{(u)} \omega_{r}^{(u)} s+\omega_{r}^{(u)^{2}}\right)\left(s^{2}+2 \zeta_{r}^{(w)} \omega_{r}^{(w)} s+\omega_{r}^{(w)^{2}}\right)\left(s P_{D}+R_{L}\right) \\
& -\sum_{r=1}^{\infty} s \hat{P}_{r}^{(w)} P_{r}^{(w)}\left(s^{2}+2 \zeta_{r}^{(u)} \omega_{r}^{(u)} s+\omega_{r}^{(u)^{2}}\right) \\
& +\sum_{r=1}^{\infty} s \hat{P}_{r}^{(u)} P_{r}^{(u)}\left(s^{2}+2 \zeta_{r}^{(w)} \omega_{r}^{(w)} s+\omega_{r}^{(w)^{2}}\right)
\end{aligned}
$$

\section{MulTimodE CEDRTL ELECTROMECHANICAL FREQUENCY RESPONSE}

Corresponding to (4) to (6), the CEDRTL model frequency response function can be formulated. In this case, after applying some simple algebra, the superposition of the piezoelectric bimorph electromechanical frequency responses can be formulated as shown in Fig. 2 to give,

$$
\mathbf{G}(j \omega)=\mathbf{H}(j \omega) \mathbf{F}(j \omega),
$$

where the FRF matrix is given by,

$$
\mathbf{H}(j \omega)=\left[\begin{array}{ll}
H_{11}(j \omega) & H_{12}(j \omega) \\
H_{21}(j \omega) & H_{22}(j \omega) \\
H_{31}(j \omega) & H_{32}(j \omega) \\
H_{41}(j \omega) & H_{42}(j \omega)
\end{array}\right],
$$

where the following output and input vector representations become,

$$
\begin{gathered}
\mathbf{G}(j \omega)=\left[\begin{array}{lll}
u_{r}(j \omega) & w_{r}(j \omega) v(j \omega) i(j \omega)
\end{array}\right]^{T}, \\
\mathbf{F}(j \omega)=\left[-\omega^{2} u_{\text {base }} e^{j \omega t}-\omega^{2} w_{\text {base }} e^{j \omega t}\right]^{T} .
\end{gathered}
$$

Each multimode FRF parameter of (9) can be formulated as shown in (12)-(15).

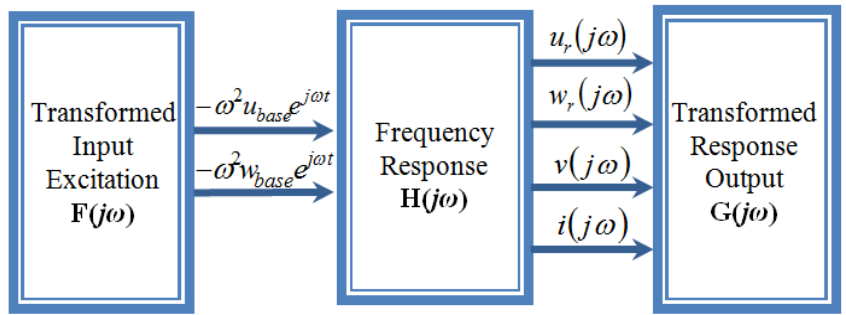

Fig. 2. Matrix superposition of multimode electromechanical frequency responses

$$
\begin{aligned}
& H_{11}(j \omega)=\left.\frac{u_{r}(j \omega)}{-\omega^{2} u_{b a s e} e^{j \omega t}}\right|_{s=j \omega}=-A_{r}^{(u)}\left(\frac{B Q_{r}^{(u)}-\sum_{r=1}^{\infty} C_{r}^{(w)} P_{r}^{(w)} Q_{r}^{(u)}}{B-\sum_{r=1}^{\infty} C_{r}^{(w)} P_{r}^{(w)}-\sum_{r=1}^{\infty} C_{r}^{(u)} P_{r}^{(u)}}\right), \quad H_{12}(j \omega)=\left.\frac{u_{r}(j \omega)}{-\omega^{2} w_{b a s e} e^{j \omega t}}\right|_{s=j \omega}=-A_{r}^{(u)}\left(\frac{\sum_{r=1}^{\infty} C_{r}^{(w)} P_{r}^{(u)} Q_{r}^{(w)}}{B-\sum_{r=1}^{\infty} C_{r}^{(w)} P_{r}^{(w)}-\sum_{r=1}^{\infty} C_{r}^{(u)} P_{r}^{(u)}}\right), \\
& H_{21}(j \omega)=\left.\frac{w_{r}(j \omega)}{-\omega^{2} u_{b a s e} e^{j \omega t}}\right|_{s=j \omega}=-A_{r}^{(w)}\left(\frac{\sum_{r=1}^{\infty} C_{r}^{(u)} P_{r}^{(w)} Q_{r}^{(u)}}{B-\sum_{r=1}^{\infty} C_{r}^{(w)} P_{r}^{(w)}-\sum_{r=1}^{\infty} C_{r}^{(u)} P_{r}^{(u)}}\right), H_{22}(j \omega)=\left.\frac{w_{r}(j \omega)}{-\omega^{2} w_{b a s e} e^{j \omega t}}\right|_{s=j \omega}=-A_{r}^{(w)}\left(\frac{B Q_{r}^{(w)}-\sum_{r=1}^{\infty} C_{r}^{(u)} P_{r}^{(u)} Q_{r}^{(w)}}{B-\sum_{r=1}^{\infty} C_{r}^{(w)} P_{r}^{(w)}-\sum_{r=1}^{\infty} C_{r}^{(u)} P_{r}^{(u)}}\right), \\
& H_{31}(j \omega)=\left.\frac{v(j \omega)}{-\omega^{2} u_{b a s e} e^{j \omega t}}\right|_{s=j \omega}=\frac{\sum_{r=1}^{\infty} C_{r}^{(u)} Q_{r}^{(u)}}{B-\sum_{r=1}^{\infty} C_{r}^{(w)} P_{r}^{(w)}-\sum_{r=1}^{\infty} C_{r}^{(u)} P_{r}^{(u)}}, H_{32}(j \omega)=\left.\frac{v(j \omega)}{-\omega^{2} w_{b a s e} e^{j \omega t}}\right|_{s=j \omega}=\frac{\sum_{r=1}^{\infty} C_{r}^{(w)} Q_{r}^{(w)}}{B-\sum_{r=1}^{\infty} C_{r}^{(w)} P_{r}^{(w)}-\sum_{r=1}^{\infty} C_{r}^{(u)} P_{r}^{(u)}}, \\
& H_{41}(j \omega)=\left.\frac{i(j \omega)}{-\omega^{2} u_{\text {base }} e^{j \omega t}}\right|_{s=j \omega}=\left.\frac{v(j \omega)}{-\omega^{2} u_{\text {base }} e^{j \omega t} R_{\text {load }}}\right|_{s=j \omega}, H_{42}(j \omega)=\left.\frac{i(j \omega)}{-\omega^{2} w_{\text {base }} e^{j \omega t}}\right|_{s=j \omega}=\left.\frac{v(j \omega)}{-\omega^{2} w_{\text {base }} e^{j \omega t} R_{\text {load }}}\right|_{s=j \omega},
\end{aligned}
$$


where :

$$
\begin{gathered}
A_{r}^{(u)}=\frac{1}{\omega_{r}^{(u)^{2}}-\omega^{2}+j 2 \zeta_{r}^{(u)} \omega_{r}^{(u)} \omega}, A_{r}^{(w)}=\frac{1}{\omega_{r}^{(w)^{2}}-\omega^{2}+j 2 \zeta_{r}^{(w)} \omega_{r}^{(w)} \omega}, \\
B=\left(j \omega P_{D}+R_{L}\right), C_{r}^{(w)}=\frac{j \omega \hat{P}_{r}^{(w)}}{\omega_{r}^{(w)^{2}}-\omega^{2}+j 2 \zeta_{r}^{(w)} \omega_{r}^{(w)} \omega}, \\
C_{r}^{(u)}=\frac{j \omega \hat{P}_{r}^{(u)}}{\omega_{r}^{(u)^{2}}-\omega^{2}+j 2 \zeta_{r}^{(u)} \omega_{r}^{(u)} \omega} .
\end{gathered}
$$

Equations (12)-(15) can be modified by transforming them back into normalised convergent eigenfunction forms [19] to obtain the FRFs as a function of position $(x)$ and frequency $(j \omega)$ on the bimorph as given in (16)-(17). Multimode FRF of power harvesting can be formulated from (18). The optimal multimode FRF of power harvesting related to the transverse excitation can be obtained by differentiating (18b) with respect to the load resistance and setting the differentiable power to zero to give the optimal load resistance as shown in (19).

$$
\begin{aligned}
& H_{11}(x, j \omega)=-\sum_{r=1}^{\infty}\left[A_{r}^{(u)} \Theta_{r}(x)\left(\frac{B Q_{r}^{(u)}-\sum_{r=1}^{\infty} C_{r}^{(w)} P_{r}^{(w)} Q_{r}^{(u)}}{B-\sum_{r=1}^{\infty} C_{r}^{(w)} P_{r}^{(w)}-\sum_{r=1}^{\infty} C_{r}^{(u)} P_{r}^{(u)}}\right)\right], H_{12}(x, j \omega)=-\sum_{r=1}^{\infty}\left[A_{r}^{(u)} \hat{\Theta}_{r}(x)\left(\frac{\sum_{r=1}^{\infty} C_{r}^{(w)} P_{r}^{(u)} Q_{r}^{(w)}}{\left.B-\sum_{r=1}^{\infty} C_{r}^{(w)} P_{r}^{(w)}-\sum_{r=1}^{\infty} C_{r}^{(u)} P_{r}^{(u)}\right)}\right],\right. \\
& H_{21}(x, j \omega)=-\sum_{r=1}^{\infty}\left[A_{r}^{(w)} \hat{\Psi}_{r}(x)\left(\frac{\sum_{r=1}^{\infty} C_{r}^{(u)} P_{r}^{(w)} Q_{r}^{(u)}}{B-\sum_{r=1}^{\infty} C_{r}^{(w)} P_{r}^{(w)}-\sum_{r=1}^{\infty} C_{r}^{(u)} P_{r}^{(u)}}\right)\right] \quad H_{22}(x, j \omega)=-\sum_{r=1}^{\infty}\left[A_{r}^{(w)} \hat{\Psi}_{r}(x)\left(\frac{B Q_{r}^{(w)}-\sum_{r=1}^{\infty} C_{r}^{(u)} P_{r}^{(u)} Q_{r}^{(w)}}{B-\sum_{r=1}^{\infty} C_{r}^{(w)} P_{r}^{(w)}-\sum_{r=1}^{\infty} C_{r}^{(u)} P_{r}^{(u)}}\right)\right], \\
& \left.\frac{P(j \omega)}{\left(-\omega^{2} u_{\text {base }} e^{j \omega t}\right)^{2}}\right|_{s=j \omega}=\left.\frac{v(j \omega)^{2}}{\left(-\omega^{2} u_{\text {base }} e^{j \omega t}\right)^{2} R_{\text {load }}}\right|_{s=j \omega},\left.\frac{P(j \omega)}{\left(-\omega^{2} w_{\text {base }} e^{j \omega t}\right)^{2}}\right|_{s=j \omega}=\left.\frac{v(j \omega)^{2}}{\left(-\omega^{2} w_{\text {base }} e^{j \omega t}\right)^{2} R_{\text {load }}}\right|_{s=j \omega}, \\
& R_{\text {load }}^{\text {opt }}=\frac{\sqrt{X(\omega)^{2}+Y(\omega)^{2}}}{X(\omega)^{2}+Y(\omega)^{2}},
\end{aligned}
$$

where:

$$
\begin{gathered}
X(\omega)=\omega P_{D}-\sum_{r=1}^{\infty} \frac{\omega \hat{P}_{r}^{(w)} P_{r}^{(w)}\left(\omega_{r}^{(w)^{2}}-\omega^{2}\right)}{\left(\omega_{r}^{(w)^{2}}-\omega^{2}\right)^{2}+\left(2 \zeta_{r}^{(w)} \omega_{r}^{(w)} \omega\right)^{2}}-\sum_{r=1}^{\infty} \frac{\omega \hat{P}_{r}^{(u)} P_{r}^{(u)}\left(\omega_{r}^{(u)^{2}}-\omega^{2}\right)}{\left.(u)^{2}-\omega^{2}\right)^{2}+\left(2 \zeta_{r}^{(u)} \omega_{r}^{(u)} \omega\right)^{2}} \\
Y(\omega)=\sum_{r=1}^{\infty} \frac{\omega \hat{P}_{r}^{(w)} P_{r}^{(w)}\left(2 \zeta_{r}^{(w)} \omega_{r}^{(w)} \omega\right)}{\left(\omega_{r}^{(w)^{2}}-\omega^{2}\right)^{2}+\left(2 \zeta_{r}^{(w)} \omega_{r}^{(w)} \omega\right)^{2}}-\sum_{r=1}^{\infty} \frac{\omega \hat{P}_{r}^{(u)} P_{r}^{(u)}\left(2 \zeta_{r}^{(u)} \omega_{r}^{(u)} \omega\right)}{\left(\omega_{r}^{(u)^{2}}-\omega^{2}\right)^{2}+\left(2 \zeta_{r}^{(u)} \omega_{r}^{(u)} \omega\right)^{2}}
\end{gathered}
$$

Corresponding to (12), (4) can be written in terms of the FRF as,

$$
u_{r}(t)=H_{11}(j \omega)\left(-\omega^{2} u_{\text {base }} \mathrm{e}^{j \omega t}\right)+H_{12}(j \omega)\left(-\omega^{2} w_{\text {base }} \mathrm{e}^{j \omega t}\right) .
$$

Modifying (20) in terms of any position along the piezoelectric beam gives,

$$
u_{r e l}(x, t)=H_{11}(x, j \omega)\left(-\omega^{2} u_{\text {base }} e^{j \omega t}\right)+H_{12}(x, j \omega)\left(-\omega^{2} w_{\text {base }} e^{j \omega t}\right) \text {. }
$$

The multimode absolute longitudinal displacement can be formulated as,

$$
\begin{aligned}
u_{a b s}(x, t) & =-\omega^{2} u_{\text {base }} \mathrm{e}^{j \omega t}+H_{11}(x, j \omega)\left(-\omega^{2} u_{\text {base }} \mathrm{e}^{j \omega t}\right) \\
& +H_{12}(x, j \omega)\left(-\omega^{2} w_{\text {base }} \mathrm{e}^{j \omega t}\right) .
\end{aligned}
$$

The generalised time dependent relative transverse displacement in (5) can be modified corresponding to (13) as, $w_{r}(t)=H_{21}(j \omega)\left(-\omega^{2} u_{\text {base }} \mathrm{e}^{j \omega t}\right)+H_{22}(j \omega)\left(-\omega^{2} w_{\text {base }} \mathrm{e}^{j \omega t}\right)$.
Corresponding to (23), the relative transverse displacement can be reformulated in terms of any position along the piezoelectric beam as,

$$
w_{r e l}(x, t)=H_{21}(x, j \omega)\left(-\omega^{2} u_{b a s e} e^{j \omega t}\right)+H_{22}(x, j \omega)\left(-\omega^{2} w_{b a s e} e^{j \alpha x}\right) .
$$

Corresponding to (24), the absolute transverse displacement can be given by,

$$
\begin{aligned}
w_{a b s}(x, t) & =-\omega^{2} w_{\text {base }} e^{j \omega t}+H_{21}(x, j \omega)\left(-\omega^{2} u_{\text {base }} e^{j \omega t}\right) \\
& +H_{22}(x, j \omega)\left(-\omega^{2} w_{\text {base }} e^{j \omega t}\right) .
\end{aligned}
$$

The generalised electrical potential can be formulated as,

$$
v(t)=H_{31}(j \omega)\left(-\omega^{2} u_{\text {base }} \mathrm{e}^{j \omega t}\right)+H_{32}(j \omega)\left(-\omega^{2} w_{\text {base }} \mathrm{e}^{j \omega t}\right) .
$$

It should be noted that $u_{\text {base }}$ and $w_{\text {base }}$ are the input base longitudinal and transverse displacement excitations on the bimorph. Corresponding to (22) and (25), (16a) and (17b) can be modified in terms of the multimode FRF of the absolute displacements and velocities relating the longitudinal and 
transverse input displacement at any position along the bimorph respectively as,

$$
\begin{aligned}
& \hat{H}_{11}^{(\text {disp })}(x, j \omega)=\frac{u_{\text {base }} e^{j \omega t}+u_{r e l}(x, t)}{-\omega^{2} u_{\text {base }} e^{j \omega t}}=-\frac{1}{\omega^{2}}+H_{11}(x, j \omega), \\
& \hat{H}_{11}^{(v e l)}(x, j \omega)=\frac{\frac{\mathrm{d}}{\mathrm{d} t}\left[u_{\text {base }} e^{j \omega t}+u_{r e l}(x, t)\right]}{-\omega^{2} u_{\text {base }} e^{j \omega t}}=\frac{1}{j \omega}+j \omega H_{11}(x, j \omega), \\
& \hat{H}_{22}^{(\text {disp })}(x, j \omega)=\frac{w_{\text {base }} e^{j \omega t}+w_{r e l}(x, t)}{-\omega^{2} w_{\text {base }} e^{j \omega t}}=-\frac{1}{\omega^{2}}+H_{22}(x, j \omega), \\
& \hat{H}_{22}^{(v e l)}(x, j \omega)=\frac{\frac{\mathrm{d}}{\mathrm{d} t}\left[w_{\text {base }} e^{j \omega t}+w_{\text {rel }}(x, t)\right]}{-\omega^{2} w_{\text {base }} e^{j \omega t}}=\frac{1}{j \omega}+j \omega H_{22}(x, j \omega) .
\end{aligned}
$$

It should be noted that (22), (25) and (27) are applicable for analysing the absolute dynamic responses and comparing the results measured using the Laser Doppler Vibrometer (LDV) at any position along the piezoelectric bimorph beam.

\section{SIMULATION AND EXPERIMENTAL RESULTS}

In this section, the trend of the first mode of the FRF was investigated by varying load resistances of $560 \Omega, 5.6 \mathrm{k} \Omega, 20$ $\mathrm{k} \Omega, 30 \mathrm{k} \Omega, 51 \mathrm{k} \Omega, 60 \mathrm{k} \Omega, 79 \mathrm{k} \Omega, 150 \mathrm{k} \Omega, 200 \mathrm{k} \Omega$ and $602 \mathrm{k} \Omega$. The bimorph input base transverse acceleration was chosen to be $3 \mathrm{~m} / \mathrm{s}^{2}$ which is equivalent to $306 \mathrm{mg}$ (1 $g=$ gravitational acceleration, $9.81 \mathrm{~m} / \mathrm{s}^{2}$ ). The results obtained were validated with an experimental study using a Laser Doppler Vibrometer (LDV) that measured the absolute velocity or displacement of the centre of the tip mass coincident with the end of the bimorph. Complete experimental setup can be seen in Fig. 3 and the properties of the bimorph and tip mass are given in Table I. We used a load resistance of $560 \Omega$, with the claim that it was approaching the short circuit resistance because at the actual short circuit condition $\left(R_{\text {load }}=0\right)$, the theoretical voltage FRF will be zero and tip absolute displacement cannot be identified. This situation cannot be used to understand the voltage and displacement FRF behaviours under electromechanical situations. If the load resistance of $1 \Omega$ was chosen, the tip absolute displacement amplitude was very close to the load resistance of $560 \Omega$. However, it was found that high level of voltage noise was present for the $1 \Omega$ experiment. Again this cannot be used to identify the voltage FRF behaviour. Therefore, we adapt the theoretical and experimental FRF studies by using $560 \Omega$ as approaching the short circuit condition. With the load resistance of $560 \Omega$, the mechanical damping ratios were identified by matching the amplitude of experimental and theoretical tip absolute displacement or velocity FRF. The physical reason by choosing damping ratios under very low

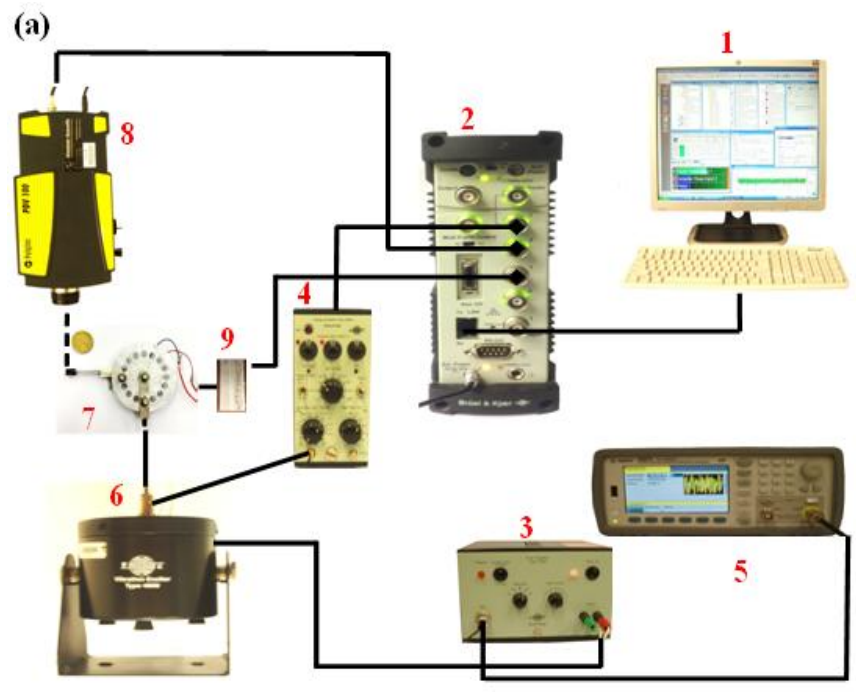

\section{(b)}

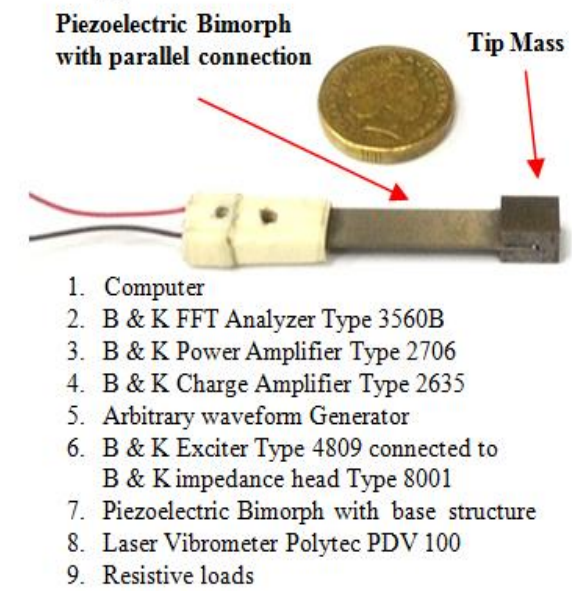

Fig. 3. (a) Experimental setup and (b) Piezoelectric bimorph beam with tip mass under parallel connection.

TABLE I

CHARACTERISTIC PROPERTIES OF THE PIEZOELECTRIC BIMORPH SYSTEM.

\begin{tabular}{lll|lcc}
\hline \hline Material properties & Piezoelectric & Brass & Geometry properties & Piezoelectric & Brass \\
\hline Young's modulus, $\bar{c}_{11}(\mathrm{GPa})$ & 66 & 105 & Length,$L(\mathrm{~mm})$ & 30.1 & 30.1 \\
Density, $\rho\left(\mathrm{kg} / \mathrm{m}^{3}\right)$ & 7800 & 9000 & Thickness, $h(\mathrm{~mm})$ & $0.19(\mathrm{each})$ & 0.13 \\
Piezoelectric constant, $d_{31}(\mathrm{pm} / \mathrm{V})$ & -190 & - & Width, $b(\mathrm{~mm})$ & 6.4 & 6.4 \\
Permittivity, $\varepsilon_{33}^{T}(\mathrm{~F} / \mathrm{m})$ & $1800 \varepsilon_{\mathrm{o}}$ & - & First coefficient $I_{\text {tip }}^{(A)}(\mathrm{kg})^{\dagger}$ & 0.0022 \\
permittivity of free space, $\varepsilon_{\mathrm{o}}(\mathrm{pF} / \mathrm{m})$ & 8.854 & - & Third coefficient $I_{\text {tip }}^{(C)}\left(\mathrm{kg} \mathrm{m}^{2}\right)^{\dagger}$ & $7.3743 \times 10^{-9}$ \\
\hline \hline
\end{tabular}

$\dagger$ Calculated according to the geometry and material properties of tip mass and the rotary inertia at centre of gravity of tip mass coincident with the end of the bimorph length. First and third coefficients refer to zeroth and second mass moment of inertias respectively. 
load resistance was to minimise the standard level of amplitude of electrical voltage FRF generated from the piezoelectric bimorph in order to approach a pure mechanical form, since mechanical damping itself was viewed as mechanical resistance behaviour due to energy losses during vibration of the piezoelectric bimorph.

At this point, it was also realised that the piezoelectric bimorph behaved as a coupled electromechanical dynamic system. Identification of mechanical damping with load resistance of $560 \Omega$ was found to provide a very close comparison between the experiment and theoretical studies. Theoretically, the energy losses can be from strain-rate (Kelvin-Voigt) and external air damping effects. However, in experimental study, damping ratios can readily be measured from the FRF without specifying the strain-rate and viscous damping effects. Moreover, once the mechanical damping ratios were determined, other FRF models with varying electrical load resistances can be plotted. The damping ratios for the transverse and longitudinal forms around the fundamental resonant frequency were found to be $\zeta_{1}^{w}=0.0139$

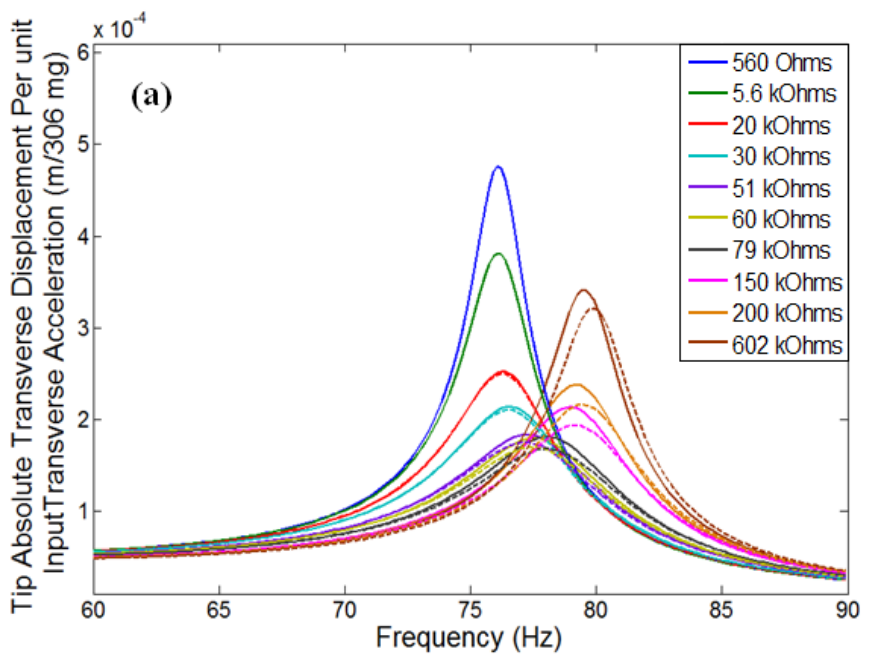

and $\zeta_{1}^{u}=0.030$. The transverse behaviour of the electromechanical system response was known to be dominant in the lower frequency range (first resonance). The effect of the longitudinal system response was also considered here from the initial strain field, contributing to the frequency response, but only with small effect. Obviously, the effect of mechanical damping ratio can be viewed as a constant value once the experimental result was taken and matched to the theoretical study. Moreover, the effect of piezoelectric coupling can further create electromechanical damping and these can also be viewed as constant values. It should be noted here that the effect of load resistance on the piezoelectric bimorph can be viewed as resistive shunt damping effect resulting in shifting of the resonant frequency with different amplitudes. In such situations, the damping effects encompassed both the mechanical and electrical forms from the electromechanical bimorph responses when the dimensional structure and material properties were chosen.

Fig. 4. Comparison between the CEDRTL (Solid line) and CEDRT (Dash line) : a) FRFs of tip absolute transverse displacement;

b) Nyquist plot of tip absolute transverse displacement.
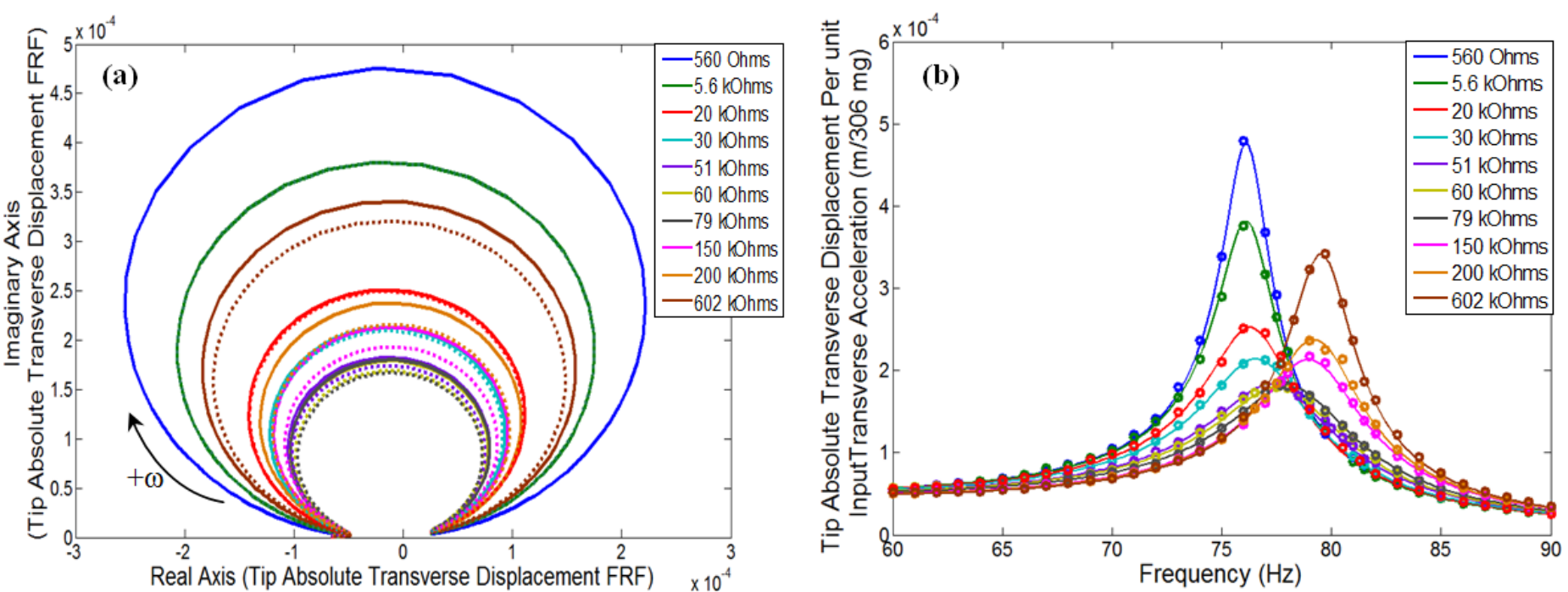

Fig. 5. Tip absolute transverse displacement responses: a) Nyquist plot of the CEDRTL (Solid line) and CEDRT (Dash line); b) FRFs of the CEDRTL (Solid line) and experimental result (Round dot). 
By considering the damping effect of the bimorph under dynamic conditions, the load resistance connected to the bimorph appeared to act as electromechanical attenuation and amplification of the amplitude across the frequency domain. Fig. 4a shows the bimorph tip transverse displacement frequency response comparison with variable load resistance.

In some cases, the effect of longitudinal extension on the frequency response can be ignored with lower load resistances $(500 \Omega, 5.6 \mathrm{k} \Omega, 20 \mathrm{k} \Omega$ and $30 \mathrm{k} \Omega$ ) because the CERDT and CERDTL responses tended to coincide with each other. However, for higher load resistances $(51 \mathrm{k} \Omega, 60 \mathrm{k} \Omega, 79 \mathrm{k} \Omega$, $150 \mathrm{k} \Omega, 200 \mathrm{k} \Omega$ and $602 \mathrm{k} \Omega$ ), the effect of longitudinal extension seemed to be more pronounced, especially at the load resistance of $602 \mathrm{k} \Omega$ where the maximum percentage difference between the two analytical models was $16 \%$. As can be seen in Fig.4b, the behaviour from the Nyquist plot changed as load resistance varied followed by the shift in frequency.

The shift in the frequency can be seen very obviously on the imaginary axis for tip absolute displacement. It is also clear

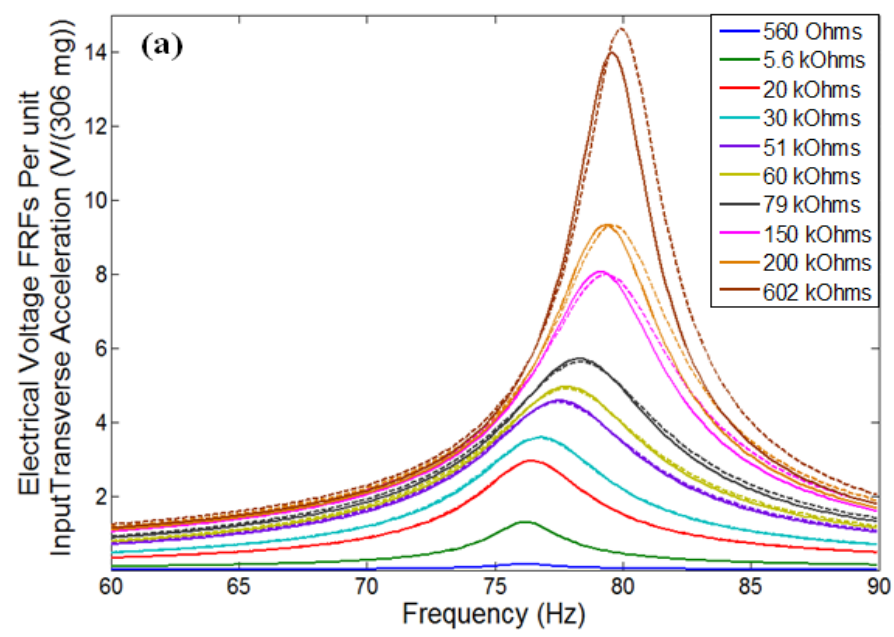

that mechanical and electromechanical dampings affect the system as seen from the Nyquist plot shown in Fig. 5a. Moreover, the FRFs of tip absolute transverse dynamic displacement with variable load resistance under the CEDRTL model seemed to be close to the experimental results as given in Fig. 5b, where the longitudinal strain-polarity field for the electromechanical dynamic response was also included here for the low frequency domain. The strain field effect used here included the transverse form with initial longitudinal strain where this affected the internal force and moment due to the transverse bending and extensional longitudinal response at each bimorph interlayer resulting in the electrical force and moment response due to the coupling effects of the piezoelectric element. Again, it can be seen from Figs.4a and $5 \mathrm{~b}$ that the first resonance frequency shifts with varying load resistance. When the load resistance tended toward short circuit at the frequency of $76.1 \mathrm{~Hz}$, the amplitude tended to give the highest value. Similar behaviour was also found at the open circuit response at the frequency of $79.6 \mathrm{~Hz}$.

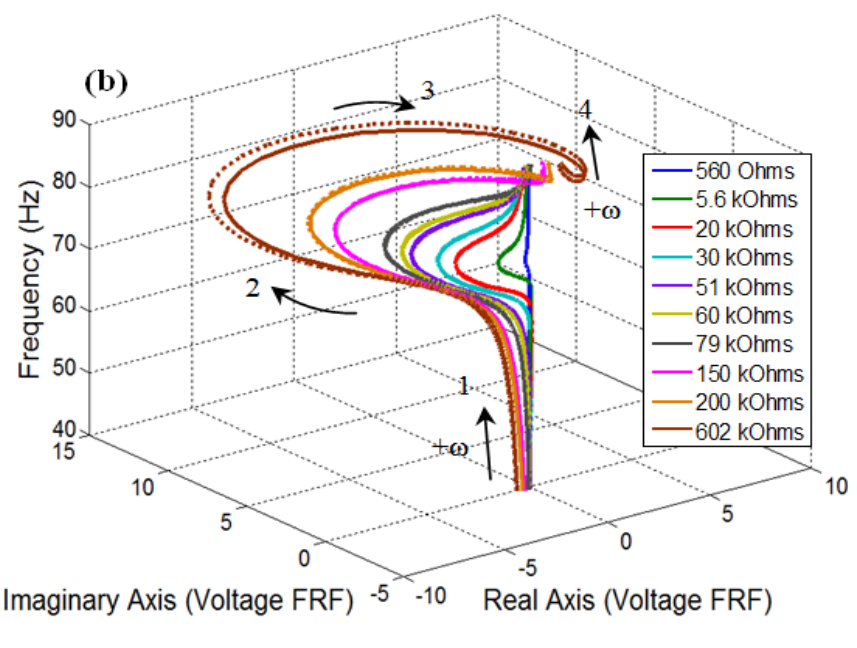

Fig. 6. Comparison between the CEDRTL (Solid line) and CEDRT (Dash line): a) FRFs of electrical voltage; b) Nyquist plot of electrical voltage FRFs.
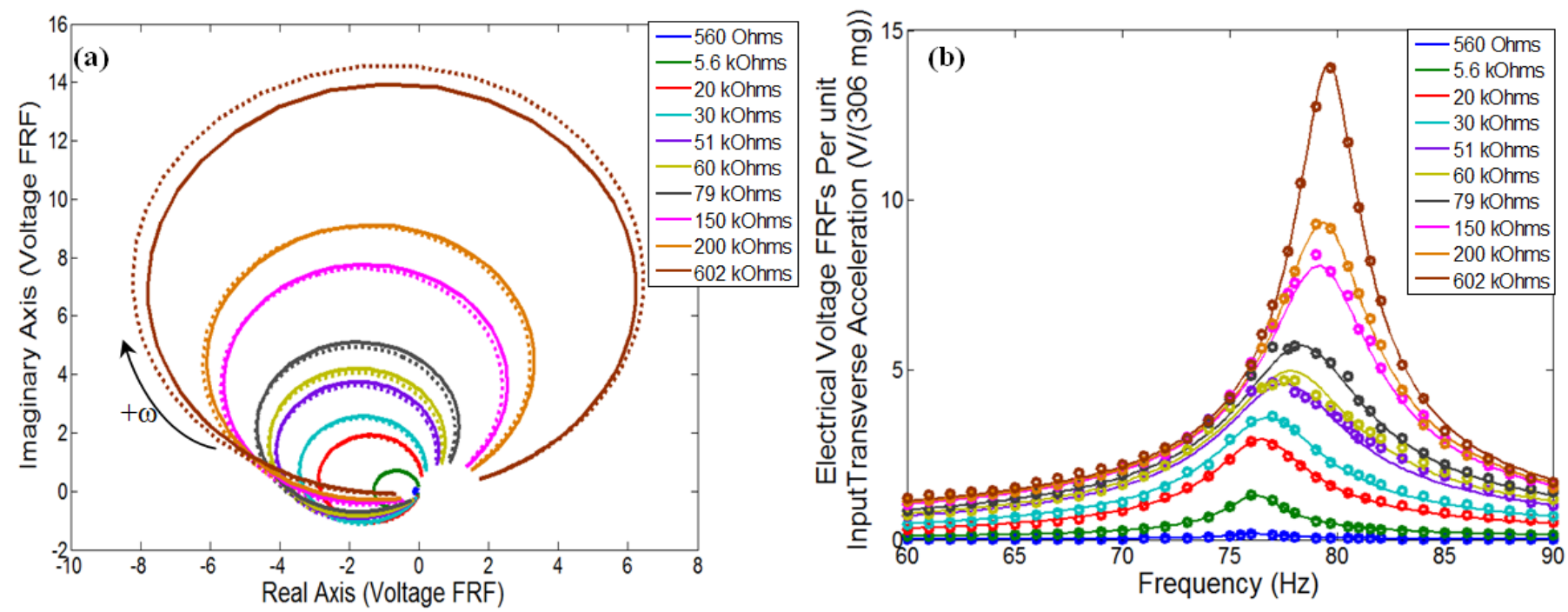

Fig. 7. Voltage responses : a) Nyquist plot of the CEDRTL (Solid line) and CEDRT (Dash line); b) FRFs of the CEDRTL (Solid line) and experimental result (Round dot). 
This indicated that the effect of the lowest and highest load resistances of $560 \Omega$ and $602 \mathrm{k} \Omega$ on the bimorph tended to reduce the sensitivity of the electrical form around the resonance frequency region. This indicates that the system is dominated by the mechanical response of the bimorph under the short and open circuits showing the highest amplitudes. Similar behaviour can also be found in the Nyquist plot in Fig. $4 \mathrm{~b}$ by viewing the imaginary and frequency axes.

Fig. 6a compares the first mode FRFs of electrical voltage of the two analytical models under varying load resistance. Both models tend to give similar trend of electrical voltage where the amplitude increased due to increasing load resistance followed by the shift in frequency. The CEDRTL response indicated a slight change when compared with the CEDRT response. The comparison between the two analytical models with the higher load resistances gave a maximum percentage difference of $22 \%$ at $602 \mathrm{k} \Omega$. In Fig. $6 \mathrm{~b}$, the difference between the two analytical methods can also be seen through the Nyquist response. By viewing the imaginary axis, the shift in frequency can be seen as load resistance changed. For this case, the Nyquist plot represented the change of FRF radius due to mechanical and electromechanical damping effects and the phase angles or arguments of FRF of $90^{\circ}-180^{\circ}$ from Fig. $7 \mathrm{~b}$ indicated variance of the FRF resonance region and the absolute amplitude values due to variable load resistance. It can also be seen that the shifting resonant response from open to short circuit load resistances started from the imaginary axis of $90^{\circ}$ to the negative real axis of $180^{\circ}$ followed by the reduction of absolute amplitude values. The comparison between the CEDRTL and experimental results were achieved with very good agreement for varying load resistance as shown in Fig. 7b. FRF of electrical current generated from the bimorph with input transverse acceleration is shown in Fig. 8a under varying load resistance. The shifting frequency due to the change of load resistance indicates a different trend compared with that shown previously. Fig. 8a shows that the comparison between the two analytical methods indicating a slight difference for some load resistances. When the load resistance approached short circuit, these analytical models overlapped for the load resistances of $560 \Omega, 5.6 \mathrm{k} \Omega$ and $20 \mathrm{k} \Omega$. There was a slight increase of electrical current amplitude with decreasing load resistance followed by decreasing resonance frequencies.
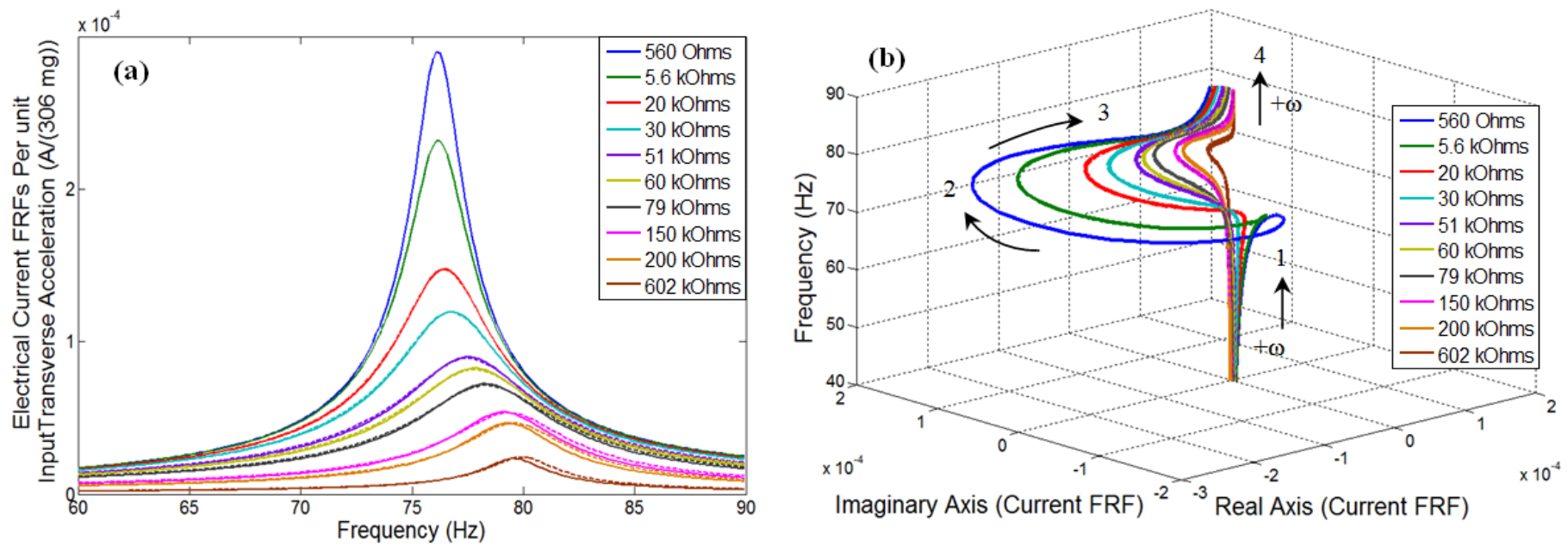

Fig. 8. Comparison between the CEDRTL (Solid line) and CEDRT (Dash line): a) FRFs of electrical current; b) Nyquist plot of electrical current FRFs.
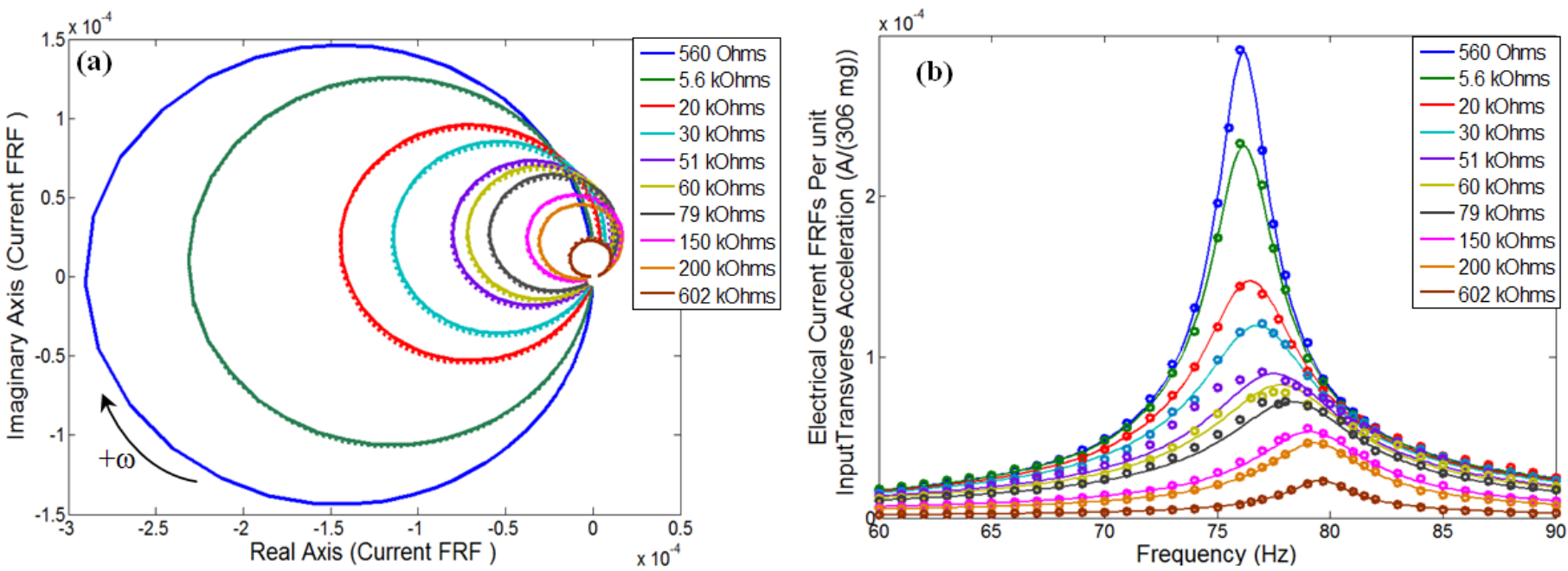

Fig. 9. Current responses : a) Nyquist plot of the CEDRTL (Solid line) and CEDRT (Dash line); b) FRFs of the CEDRTL (Solid line) and experimental result (Round dot). 
In this case, the electrical current frequency response with varying load resistance shows the electromechanical attenuation behaviour. The trend of electrical current indicates opposite behaviour from the electric voltage response as shown in Fig. 6a.

The CEDRTL and experimental results gave very good agreement under varying load resistances as shown in Fig. 8b. Furthermore, there was a slightly different trend in the specific current amplitude under the higher load resistances between the two analytical responses with the maximum percentage difference of $22 \%$ observed within the non-resonance regions. The similar behaviour can be seen from the Nyquist plot in Fig. 8b where the response changed as the open circuit load resistance moved to short circuit load resistance. As can be seen in Fig. $8 \mathrm{~b}$ and $9 \mathrm{a}$, the shifting resonance based on the increasing absolute amplitude values started from the positive imaginary axis of $90^{\circ}$ to the negative real axis of $180^{\circ}$. The electrical power harvesting frequency response of the bimorph is presented for varying load resistance. As can be seen from Fig. 10a, the comparison between the two analytical response models was shown to yield slightly different amplitudes with a maximum percentage difference being $49 \%$ for the offresonance regions with the higher load resistance approaching open circuit.

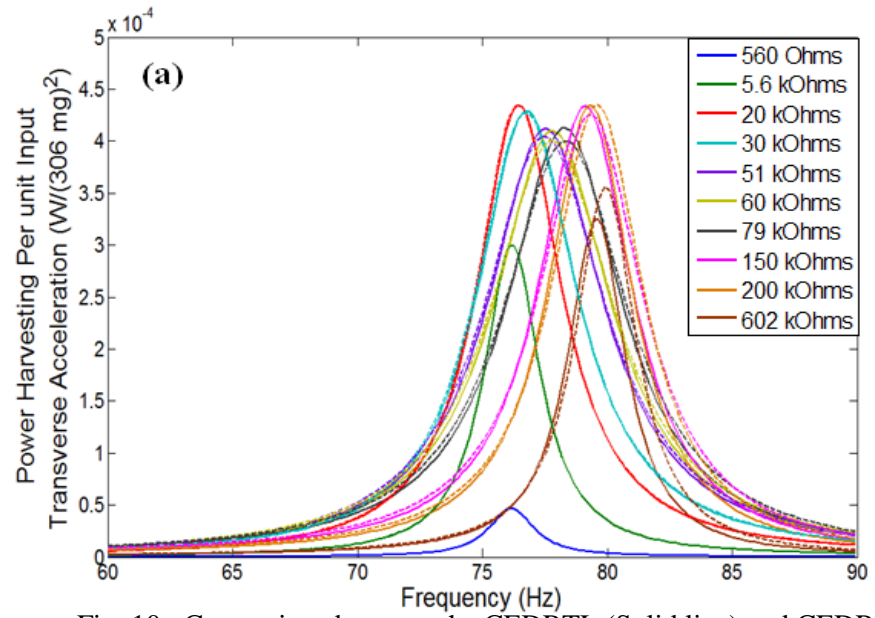

Fig. 10. Comparison between the CEDRTL (Solid line) and CEDRT power FRFs.
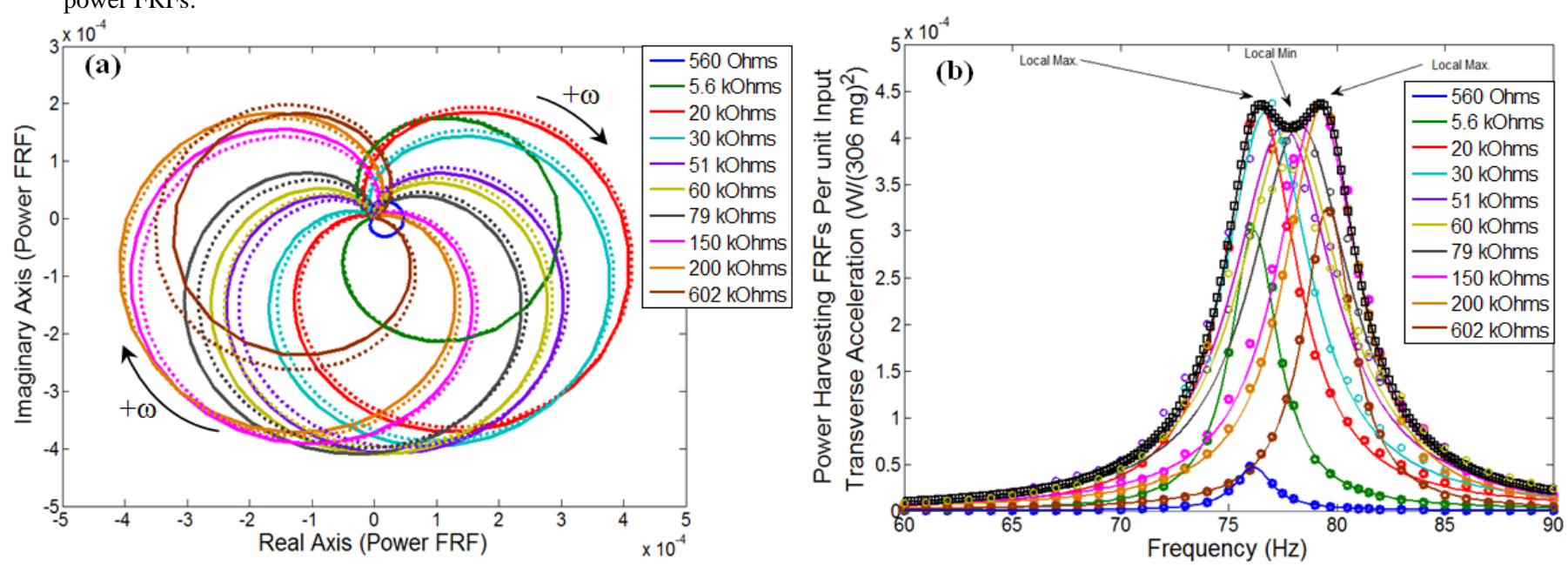

Fig. 11. Power responses: a) Nyquist plot of the CEDRTL (Solid line) and CEDRT (Dash line); b) FRFs of the CEDRTL (Solid line) and experimental result (Round dot).
Moreover, the trend of the power harvesting FRF tended to give different results when compared with previous cases. Another important aspect which can be reported here is that distribution of load resistances from short to open circuits tended to form symmetrical pattern as shown in the Nyquist circle from Fig. 10b. Both analytical methods seemed to give different amplitudes. In this case, the clearer view can be seen in Fig. 11a where the shifting resonant frequencies based on the increasing absolute amplitude values were distributed on the Nyquist plot with phase angles from negative real axis of $180^{\circ}$ to positive real axis of $360^{\circ}$ due to the change of resistance.

In terms of the Nyquist response, the trend of power harvesting depends not only on the varying load resistances but also on the chosen properties of the piezoelectric layers and the geometry of the bimorph model. For example; even though the geometry of the bimorph was chosen with the same parameters, it can still have different physical properties like capacitance and piezoelectric coupling resulting in different power harvesting values. This indicates that the chosen load resistances need to be investigated first to show the pattern of frequency response around the first mode.

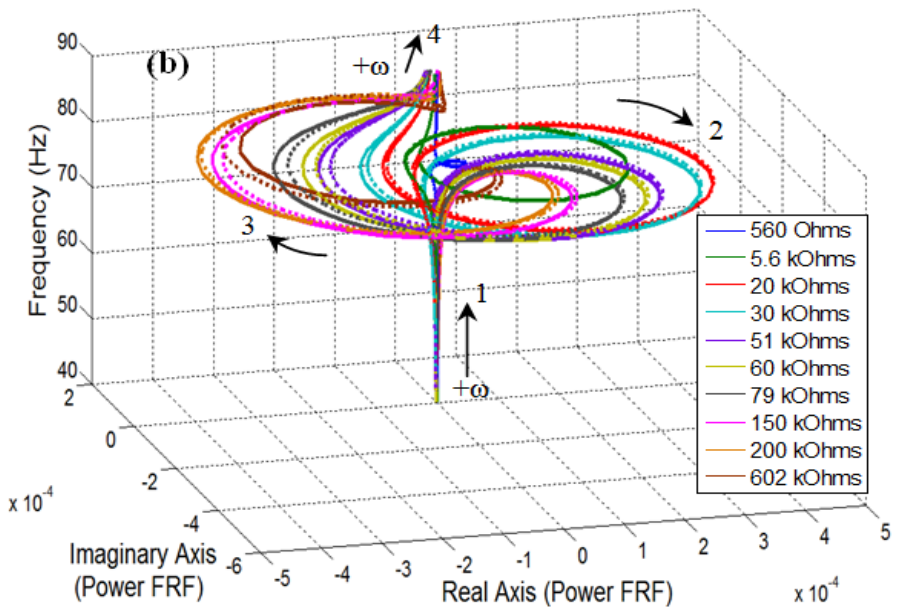

(Dash line): a) FRFs of electrical power; b) Nyquist plot of electrical 
By comparing the different cases of FRFs like the tip absolute displacement, electrical current and voltage amplitudes; the short and open circuit resonance frequencies seemed to give different trends. For example, the trend of electric current response as a function of load resistance can be compared with the previous trends of tip absolute displacement and electric voltage.

The tip absolute displacement is shown to have the highest amplitudes with the load resistance approaching short and open circuits. However, the voltage amplitude increased from short to open circuit resistance whereas the increase of electrical current amplitude occurred from the open to short circuits. Therefore, the highest displacement is not a substantial basis for providing the maximum current and maximum voltage. This indicates that open and short circuit load resistances are unsuitable for power harvester optimisation as the highest tip absolute displacement amplitude did not result in the highest power harvesting. In fact, both the short and open circuit resistances indicated the lowest value of power amplitudes. When considering optimal load resistances, three local points of load resistances were observed. The optimal power can be obtained from the black square curve of Fig. $11 \mathrm{~b}$. At this case, with the input base excitation of $0.306 \mathrm{~g}$, the local maximum power at load resistance of $20 \mathrm{k} \Omega$ and $200 \mathrm{k} \Omega$ can be seen to coincide with the optimal curve with the amplitude of $0.43 \mathrm{~mW}$, whereas a local minimum power of $0.41 \mathrm{~mW}$ at load resistance $60 \mathrm{k} \Omega$ also overlapped with an intermediate optimal curve as shown in Fig.11b. This local point indicated the lowest tip absolute displacement amplitude around the resonance frequency but showed convenient values for voltage and current. When the optimal local power density point was considered along with the active volume of the piezoelectric bimorph, the power density of the bimorph became $4.17 \mathrm{~mW} / \mathrm{cm}^{3}$. By considering the Nyquist circle as shown in Fig. 11a, two local maximum powers at load resistances of $200 \mathrm{k} \Omega$ and $20 \mathrm{k} \Omega$ can also be obtained at Nyquist phase angles of $215^{\circ}$ and $300^{\circ}$ respectively. However, local minimum power at load resistance of $60 \mathrm{k} \Omega$ also indicated the optimal value was located at the predominant negative imaginary axis at $270^{\circ}$.

\section{CONCLUSION}

This paper presented results obtained from CFBV analytical modelling and experimental measurements of the electromechanical dynamic response of a piezoelectric bimorph beam with tip mass. The results from the two analytical models were plotted under various load resistance values from short circuit to open circuit. The CEDRTL model demonstrates the electromechanical principle where the strain field includes the coupling between the transverse bending and longitudinal extension forms, whereas the CEDRT model ignored the longitudinal effect. The mechanical damping ratios were determined by matching the frequency amplitudes at the short circuit load resistance from the experimental results with the theoretical studies. The shifting of the resonance frequencies occurred as the load resistances changed where this case mostly occurred at the first mode. The Nyquist frequency response of tip absolute displacement, voltage, current and power gave different patterns where the imaginary and real axis amplitudes were used to illustrate the frequency response behaviours. Instead of mechanical and electromechanical dampings, the change of phase angles and amplitude values in the Nyquist plot also indicated change of resonant frequency due to load resistance viewed as resistive shunt damping. Moreover, the piezoelectric bimorph beams with the tip mass under the input base transverse excitation using two analytical models have been compared to show the agreement with the experimental results. The two analytical model comparisons gave slight changes of amplitude trends especially where the load resistances approached the open circuit condition. However, when the load resistance approached the short circuit, the frequency amplitudes given from two analytical models seemed to overlap each other. The FRFs from the CEDRTL model and the experimental results of the bimorph with the tip mass have been compared with good agreement. The effect of longitudinal response under lower load resistance can be ignored, as the two analytical models overlapped each other. However, the longitudinal response on the bimorph with the higher load resistance may not be ignored, especially when the tip mass is included with two input base excitations.

All results showed the frequency response functions of tip absolute dynamic displacement, electrical voltage, current and power. The increasing tip absolute displacement occurred when load resistances approached short and open circuits. The maximum displacement amplitude in the Nyguist plot dominantly occurred in the positive imaginary axis. The electrical voltage FRF shifted from short to open circuits resulting in increasing amplitudes. The Nyquist response also showed the change in maximum electrical voltage amplitudes from negative real axis to positive imaginary axis followed by the change of load resistance from short to open circuits. In the opposite behaviour from voltage, the electrical current amplitude reached the maximum level as the load resistance approached short circuit resulting the shift in resonant frequency to a higher value. The electrical current Nyquist response also showed opposite behaviour from voltage where the maximum amplitude increased from positive imaginary axis to negative real axis. Moreover, the power FRF at load resistances approaching short and open circuits did not result in maximum amplitudes. The identification of optimal resistances has assisted in finding the maximum power. In the Nyquist response, the power amplitudes were distributed from the negative real axis of $180^{\circ}$ to the positive real axis of $360^{\circ}$.

The bimorph system, when used in vibration environments with rotating equipment, will be subject to multidirectional input excitations. This will normally result in coupled bending and longitudinal input and subsequently coupled piezoelectric response. Moreover, this analysis can provide benefit for design of power conditioning electronic circuits in the application of future portable power harvesting devices for smart sensor wireless applications.

\section{APPENDIX}

A. Mass Moment of Inertias of the Piezoelectric Bimorph and Tip Mass 
The zeroth mass moment of inertia of the piezoelectric bimorph was given as,

$$
\hat{I}^{(A)}=b h_{p} \rho^{(1)}+b h_{s} \rho^{(2)}+b h_{p} \rho^{(3)} .
$$

The coefficients $\rho^{(1)}=\rho^{(3)}$ and $\rho^{(2)}$ represent densities of upper and lower piezoelectric and middle brass layers, respectively. Coefficients $b, h_{p}$, and $h_{s}$ indicate the bimorph width, piezoelectric and brass thicknesses, respectively. The zeroth mass moment of inertia of the proof mass based on Fig. 1 can also be formulated as,

$$
I_{t i p}^{(A)}=\left(h_{t i p} l_{t i p}-\left(2 h_{p}+h_{s}\right) l_{o}\right) s_{t i p} \rho_{t i p}^{(A)}
$$

The second mass moment of inertia or rotary inertia at the centre of gravity of the proof mass can be formulated as,

$$
\begin{aligned}
I_{\text {tip }}^{(C)} & =\left\{\left(\frac{\left(l_{t i p}^{2}+h_{t i p}^{2}\right)}{12}+\bar{x}_{1}{ }^{2}-\frac{\left(\left(2 h_{p}+h_{s}\right)^{2}+l_{o}^{2}\right)}{12}-\bar{x}_{2}{ }^{2}\right)\right. \\
& \left.\times\left(l_{t i p} h_{t i p}-\left(2 h_{p}+h_{s}\right) l_{o}\right)\right\} s_{t i p} \rho_{t i p}^{(C)},
\end{aligned}
$$

where $\bar{x}_{I}=x_{g}-l_{t i p} / 2, \bar{x}_{2}=x_{g}-l_{o} / 2$. Other coefficients $x_{g}$, $s_{\text {tip }}, h_{\text {tip }}$, and $l_{\text {tip }}$ indicate the centre of gravity, width, height, and length of geometry of the tip mass. It is noted that equation (A3) can also be seen in (D6).

\section{B. Stiffness Coefficients for the Piezoelectric Bimorph} Interlayer

The extensional stiffness coefficient can be formulated as,

$$
\hat{C}^{(D)}=b h_{p} \bar{c}_{11}^{(1)}+b h_{s} \bar{c}_{11}^{(2)}+b h_{p} \bar{c}_{11}^{(3)},
$$

The transverse stiffness coefficient can be formulated as,

$$
\begin{aligned}
\hat{C}^{(F)}=\left(-\frac{h_{s}{ }^{3}}{24}-\right. & \left.\frac{1}{3}\left(-h_{p}-\frac{h_{s}}{2}\right)^{3}\right) b \bar{c}_{11}^{(1)}+\frac{b h_{s}{ }^{3} \bar{c}_{11}^{(2)}}{4} \\
& +\left(\frac{1}{3}\left(h_{p}+\frac{h_{s}}{2}\right)^{3}-\frac{h_{s}{ }^{3}}{24}\right) b \bar{c}_{11}^{(3)} .
\end{aligned}
$$

where $\bar{c}_{11}^{(1)}=\bar{c}_{11}^{(3)}=\bar{c}_{11}^{E}$ and $\bar{c}_{11}^{(2)}$ represent elastic stiffnesses of the piezoelectric layers at constant electric field and the brass layer, respectively. It is noted that equations (B1) and (B2) can also be seen in (D4) and (D8), respectively.

\section{Forward and Backward Piezoelectric Coupling} Coefficients and Internal Capacitance of Piezoelectric Bimorph

It is noted that forward and backward piezoelectric couplings $\hat{\vartheta}$ come from the converse and direct effect of the piezoelectric material respectively, but indicate the same result [19].

\section{Bimorph Series Electrical Connection}

a) Piezoelectric coupling due to transverse bending can be formulated as,

$$
\hat{\vartheta}^{(H)}=-\frac{b e_{31}^{(1)}}{2 h_{p}}\left(\frac{h_{p}{ }^{2}}{2}+\frac{h_{p} h_{s}}{2}\right)-\frac{b e_{31}^{(3)}}{2 h_{p}}\left(\frac{h_{p}{ }^{2}}{2}+\frac{h_{p} h_{s}}{2}\right)=-\frac{b e_{31}}{h_{p}}\left(\frac{h_{p}{ }^{2}}{2}+\frac{h_{p} h_{s}}{2}\right) .
$$

b) Piezoelectric coupling due to longitudinal extension can also be stated as,

$$
\hat{\vartheta}^{(G)}=\frac{b h_{p} e_{31}^{(1)}}{2 h_{p}}+\frac{b h_{p} e_{31}^{(3)}}{2 h_{p}}=b e_{31} .
$$

The capacitance of the piezoelectric element was calculated as,

$$
\hat{C}_{p}=\frac{b h_{p} \varepsilon_{33}^{(1)}}{4 h_{p}{ }^{2}}+\frac{b h_{p} \varepsilon_{33}^{(3)}}{4 h_{p}{ }^{2}}=\frac{b \varepsilon_{33}^{S}}{2 h_{p}} .
$$

Since the upper and lower layers of the piezoelectric bimorph indicated the same material and geometrical structure, the permittivity of the piezoelectric element will be the same where $\varepsilon_{33}^{(1)}=\varepsilon_{33}^{(3)}=\varepsilon_{33}^{S}$. It should be noted that $\varepsilon_{33}^{S}$ is the permittivity at constant strain that can be further formulated as $\varepsilon_{33}^{S}=\varepsilon_{33}^{T}-e_{31} d_{31}$ or $\varepsilon_{33}^{S}=\varepsilon_{33}^{T}-d_{31}{ }^{2} \bar{c}_{11}^{E}$ where $\bar{c}_{11}^{E}=1 / s_{11}^{E}, \varepsilon_{33}^{T}$ is the permittivity at constant stress and $s_{11}^{E}$ is the elastic compliance at constant electric field.

\section{Bimorph Parallel Electrical Connection}

a) Piezoelectric coupling due to transverse bending can be formulated as,

$$
\hat{\vartheta}^{(H)}=-\frac{e_{31}^{(1)}}{h_{p}}\left(\frac{h_{p}{ }^{2}}{2}+\frac{h_{p} h_{s}}{2}\right)-\frac{e_{31}^{(3)} b}{h_{p}}\left(\frac{h_{p}{ }^{2}}{2}+\frac{h_{p} h_{s}}{2}\right)=-\frac{2 e_{31} b}{h_{p}}\left(\frac{h_{p}{ }^{2}}{2}+\frac{h_{p} h_{s}}{2}\right) .
$$

b) Piezoelectric coupling due to longitudinal extension can be formulated as,

$$
\hat{\vartheta}^{(G)}=b e_{31}^{(1)}+b e_{31}^{(3)}=2 b e_{31} .
$$

The capacitance of the piezoelectric element for parallel connection was given by,

$$
\hat{C}_{p}=\frac{b h_{p} \varepsilon_{33}^{(1)}}{h_{p}{ }^{2}}+\frac{b h_{p} \varepsilon_{33}^{(3)}}{h_{p}{ }^{2}}=\frac{2 b \varepsilon_{33}^{S}}{h_{p}} .
$$

\section{Mode shapes of the piezoelectric bimorph beam with tip} mass

The mode shape of longitudinal form can be formulated as,

$$
\Theta_{r}(x)=b_{1 r} \sin \gamma x \text {. }
$$

Since equation (D1) contains variable $b_{1 r}$ as the longitudinal amplitude constant, the normalised mode shapes can be formulated as,

$$
\hat{\Theta}_{r}(x)=\frac{\Theta_{r}(x)}{\left(\int_{0}^{L} \hat{I}^{(A)} \Theta_{r}(x)^{2} \mathrm{~d} x+I_{\text {tip }}^{(A)} \Theta_{r}(L)^{2}\right)^{1 / 2}} r=1,2, \ldots ., m .
$$

In terms of orthonormalisation, equation (D2) should meet the specific orthogonality condition based on the boundary condition as,

$$
\begin{gathered}
\int_{0}^{L} \hat{I}^{(A)} \hat{\Theta}_{r}(x) \hat{\Theta}_{q}(x) \mathrm{d} x+I_{t i p}^{(A)} \hat{\Theta}_{r}(L) \hat{\Theta}_{q}(L)=\delta_{r q}, \\
\int_{0}^{L} \hat{C}^{(D)} \frac{\mathrm{d} \hat{\Theta}_{r}(x)}{\mathrm{d} x} \frac{\mathrm{d} \hat{\Theta}_{q}(x)}{\mathrm{d} x} \mathrm{~d} x=\omega_{r}^{(u)^{2}} \delta_{r q},
\end{gathered}
$$

where $\delta_{r q}$ is the Kronecker delta, defined as unity for $q=r$ and zero for $q \neq r$. The mode shape of transverse bending can be formulated as,

$$
\Psi_{r}(x)=c_{1 r}\left(\cos \mu x-\cosh \mu x+\frac{A_{21}}{A_{22}}(\sin \mu x-\sinh \mu x)\right),
$$


where:

$$
\begin{aligned}
& A_{21}=(\sin \mu L-\sinh \mu L)+\frac{I_{t i p}^{(A)} \mu}{\hat{I}^{(A)}}(\cos \mu L-\cosh \mu L), \\
& A_{22}=(\cos \mu L+\cosh \mu L)-\frac{I_{t i p}^{(A)} \mu}{\hat{I}^{(A)}}(\sin \mu L-\sinh \mu L) .
\end{aligned}
$$

Since equation (D4) contains variable $c_{1 r}$ as the transverse amplitude constant, the normalised mode shape can be formulated as,

$$
\begin{aligned}
& \hat{\Psi}_{r}(x)=\frac{\Psi_{r}(x)}{\left(\int_{0}^{L} \hat{I}^{(A)} \Psi_{r}(x)^{2} \mathrm{~d} x+I_{\text {tip }}^{(A)} \Psi_{r}(L)^{2}+I_{\text {tip }}^{(C)}\left(\frac{\mathrm{d} \Psi_{r}}{\mathrm{~d} x}(L)\right)^{2}\right)^{1 / 2}}, \\
& r=1,2, \ldots, m \text {. }
\end{aligned}
$$

In terms of orthonormalisation, equation (D5) should meet the specific orthogonality property of the mechanical dynamic equations based on the boundary condition as,

$$
\begin{gathered}
\int_{0}^{L} \hat{I}^{(A)} \hat{\Psi}_{r}(x) \hat{\Psi}_{q}(x) \mathrm{d} x+I_{\text {tip }}^{(A)} \hat{\Psi}_{r}(L) \hat{\Psi}_{q}(L)+I_{t i p}^{(C)} \frac{\mathrm{d} \hat{\Psi}_{r}(L)}{\mathrm{d} x} \frac{\mathrm{d} \hat{\Psi}_{q}(L)}{\mathrm{d} x}=\delta_{r q}, \\
\int_{0}^{L} \hat{C}^{(F)} \frac{\mathrm{d}^{2} \hat{\Psi}_{r}(x)}{\mathrm{d} x^{2}} \frac{\mathrm{d}^{2} \hat{\Psi}_{q}(x)}{\mathrm{d} x^{2}} \mathrm{~d} x=\omega_{r}^{(w)} \delta_{r q} .
\end{gathered}
$$

\section{REFERENCES}

[1] S. Priya, "Advances in energy harvesting using low profile piezoelectric transducers," J. Electroceramic, vol. 19, pp.167-184, 2007.

[2] H. A. Sodano, D. J. Inman, and G. Park, "A review of power harvesting from vibration using piezoelectric materials," Shock Vib. Dig., vol. 36, pp. 197-205, May 2004.

[3] K.A. Cook-Chennault, N. Thambi, M.A. Bitetto and E.B. Hameyie, "Piezoelectric energy harvesting," Bullet. Sci.Technol. Soc., vol. 28, pp. 496-509, 2008.

[4] R.J.M. Vullers, R. van Schijk, C. Doms, I., van Hoof and R. Meterns, "Micropower energy harvesting,"Solid-State Electro., vol. 53, pp. 684693, 2009.

[5] P. C.-P. Chao, "Energy harvesting electronics for vibratory devices in self-powered sensors,"IEEE Sensor J., vol. 11, no. 12, pp. 3106-3121, 2011.

[6] S. Roundy and P.K. Wright, "A piezoelectric vibration based generator for wireless electronics," Smart Mater. Struct., vol. 18, pp. 1131-1142, 2004

[7] J.C. Park, J.Y. Park, and Y-P Lee, "Modeling and Characterization of Piezoelectric d33-Mode MEMS Energy Harvester," IEEE/ASME $J$. Microelectromech. Syst., vol. 19, pp. 1215-1222, 2010.

[8] J. Liang and W.-H Liao, "Impedance modeling and analysis for piezoelectric energy harvesting Systems," IEEE/ASME Trans. Mechatronic, vol. 17, no. 6, pp. 1145-1157, 2012.

[9] H. Takeda, K. Mihara, T. Yoshimura, T.Hoshina, and T. Tsurumi, "Effect of material constants on power output in piezoelectric vibration-based generators," IEEE Trans. Ultrason. Ferroelectr. Freq. Contr., vol. 58, no. 9, pp. 1852-1859, 2011.

[10] A. Mathers, K.S. Moon, J. Yi, "A Vibration-based PMN-PT energy harvester," IEEE Sensor J., vol. 9, no. 7, pp. 731-739, 2009.

[11] A. M. Wickenheiser, T. Reissman, W.-J. Wu, and E. Garcia, "Modeling the effects of electromechanical coupling on energy storage through piezoelectric energy harvesting, "IEEE/ASME Trans. Mechatronic, vol. 15, no. 3, pp.400-411, 2010.

[12] N. E. duToit, B. L.Wardle, and S.Kim, "Design considerations for MEMS scale piezoelectric mechanical vibration energy harvesters," $J$. Integr. Ferroelectr., vol. 71, pp. 121-160, 2005.

[13] M. Kim, M. Hoegen, J. Dugundji, B.L. Wardle, "Modeling and experimental verification of proof mass effects on vibration energy harvester performance," Smart. Mater. Struct., vol. 19, art. no. 045023, 2010 .
[14] F. Goldschmidtboeing, P. Woias, "Characterization of different beam shapes for piezoelectric energy harvesting," J. Micromech. Microeng, vol. 18, art. no.104013, 2008.

[15] R. Ly, M. Rguitia, S. D’Astorga, A. Hajjaji, C. Courtoisa, A. Lerichea, "Modeling and characterization of piezoelectric cantilever bending sensor for energy harvesting," Sens.Actuators A, vol. 168, pp. 95-100, 2011.

[16] M.F. Lumentut, K.K. Teh and I.M. Howard, "Transient vibration analysis for generating low electrical power," Proc.14 ${ }^{\text {th }}$ Int. Cong. Sound Vib., Cairns, Australia, Jul. 2007.

[17] M.F. Lumentut, K.K. Teh and I.M. Howard, "Computational FEA model of a coupled piezoelectric sensor and plate structure for energy harvesting," Aust. J. Mech. Eng., vol. 5, pp. 199-208, 2008.

[18] M.F. Lumentut and I.M. Howard, "The experimental validation of an electromechanical dynamic model of a piezoelectric bimorph beam for prediction of power generation," Proc. $6^{\text {th }}$ Aust. Cong. App. Mech., pp. 908-917, Dec. 2010.

[19] M.F. Lumentut and I.M. Howard, "Analytical Modeling of self-powered electromechanical piezoelectric bimorph beams with multidirectional excitation," Int. J. Smart Nano Mater., vol. 2, no.3, pp. 134-175, 2011.

[20] M.F. Lumentut and I.M. Howard, "Analytical and experimental Comparisons of electromechanical vibration response of a piezoelectric bimorph beam for power harvesting," Mech. Syst. Signal Proc., [Online]. Available: http://dx.doi.org/10.1016/j.ymssp.2011.07.010.

[21] IEEE Standard on Piezoelectricity, ANSI/IEEE Std. 176, 1987.

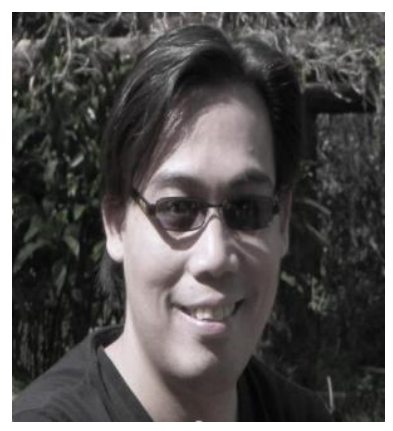

Mikail F. Lumentut received a M.Phil degree in numerical methods of smart structures and a Ph.D degree in electromechanics from Curtin University of Technology, Perth, Australia, in 2006 and 2010, respectively. Since November 2010, he has been a research assistant in the Department of Mechanical Engineering, Curtin University of Technology.

His research interests include experimental technique, analytical vibration modelling, nonlinear dynamics, finite element analysis, microelectromechanical system dynamics, mechanics of smart material and structure, robust control system, aeroelastic flutter dynamics, and energy harvesting.

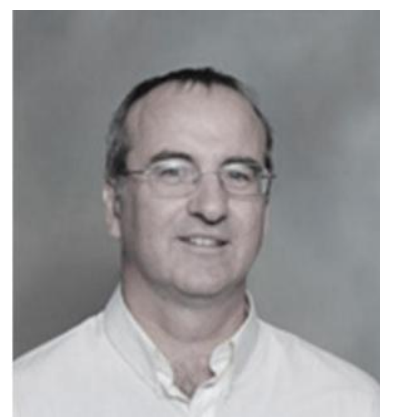

Ian M. Howard became a member of Engineers Australia in 1994. He graduated with BE (Hons), mechanical engineering and $\mathrm{Ph} . \mathrm{D}$ structural dynamics, from the University of Western Australia, Perth, Australia, in 1984 and 1988 respectively.

He was employed with Defence Science and Technology Organisation (DSTO) at the Aeronautical Research Laboratory, Melbourne, Australia from 1988 to 1994 as a Research Scientist studying helicopter gearbox vibration health monitoring. He has worked at Curtin University in Perth, Australia since 1994, in the Department of Mechanical Engineering, being promoted to A/Prof in 2005.

His research career has largely focused on the measurement, modelling and signal processing of vibration for the detection of incipient failure of rotating machinery, primarily with gears and bearings. He currently supervises several postgraduate students in areas of reliability, dynamic modeling and smart sensor power harvesting, as well as teaching undergraduate courses in Machine Dynamics, Vibration and Noise.

A/Prof Howard has served on the National Committee of Applied Mechanics within Engineers Australia since 2003, currently being the committee chair. He has been an external reviewer for various journals including Journal of Sound and Vibration, ASME Journal of Mechanical Design, Mechanical Systems and Signal Processing, Institute of Physics Smart Materials and Structures and Applied Soft Computing. 